\title{
Détermination indirecte des limites d'Atterberg par gravimétrie dynamique
}

\section{Determination of Atterberg limits by dynamic gravimetry.}

\author{
Chantal Proust ${ }^{\underline{a}}$ Agnès Jullien ${ }^{\underline{b}}$ and Lydie Le Forestier ${ }^{\underline{a}}$, \\ aISTO (UMR 6113), Polytech' Orléans, université d'Orléans, 45072 Orléans cedex 2, France \\ ${ }^{\mathrm{b}}$ Laboratoire central des ponts et chaussées, route Bouaye, BP 4129, 44341 Bouguenais cedex, \\ France
}

\section{Résumé}

Ce travail présente une nouvelle méthode de mesure des limites d'Atterberg par dessiccation dynamique. En effet, pour certaines argiles polluées ou des mélanges d'argiles, les méthodes classiques ne peuvent pas être mises en œuvre. Le principe de détermination des limites de liquidité $\left(W_{\mathrm{L}}\right)$ et plasticité $\left(W_{\mathrm{P}}\right)$ par cette méthode de dessiccation est présenté. Les valeurs des limites d'Atterberg obtenues sur des argiles courantes se révèlent peu différentes de celles déterminées par la méthode de Casagrande. Ces résultats permettent de considérer cette méthode comme une caractérisation physique des boues argileuses.

\section{Abstract}

This study presents a new method for Atterberg limits measurements using dynamic drying. Indeed, for some polluted clays, or clay mixtures, classical methods cannot be used. The principle of liquid $\left(W_{\mathrm{L}}\right)$ and plasticity $\left(W_{\mathrm{P}}\right)$ limits determination by this method is presented. Experimental values for classical clays are close to those obtained with Casagrande's methods, allowing for larger physical mud characterisation.

Mots-clés: limites d'Atterberg; argile; dessiccation; smectite; kaolinite Keywords: Atterberg limits; clay; drying method; smectite; kaolinite

\section{Introduction}

Les travaux de recherche sur le comportement des argiles ont progressivement évolué, au cours des dernières décennies, de leur caractérisation mécanique et hydraulique en laboratoire à l'état saturé vers leur caractérisation à l'état non saturé, donnant lieu au développement de la modélisation constitutive et du transfert de masse d'eau à l'état non saturé. Cette évolution a été favorisée par les besoins de modélisation identifiés dans le cadre de l'étude des ouvrages de stockage de déchets radioactifs à grande profondeur. Ainsi, il est admis aujourd'hui d'aborder le comportement mécanique des argiles non saturées par l'utilisation de tenseurs capillaires [6], voire en modifiant leurs lois de comportement mécanique, pour obtenir des lois constitutives valables dans le domaine non saturé et jusqu'à saturation [1]. Ainsi, un régime non saturé (écrouissage hydrique) induit une plus faible plasticité de l'argile. 
Plus récemment, la modélisation des effets couplés chimie-transport a également progressé, bien que le problème de l'écrouissage chimique des argiles sous l'effet de polluants soit un domaine très peu étudié sur le plan mécanique, tant au niveau des lois de comportement que des limites de liquidité [4] and [5]. C'est cet ensemble de considérations qui a conduit à utiliser un appareillage de dessiccation d'échantillons de boues pour déterminer des limites de liquidité des matériaux argileux. Des essais ont été réalisés sur des argiles classiques pour valider la méthode et permettre l'étude ultérieure de matériaux non standard ou de boues polluées. Dans ce dernier cas, au vu du grand nombre de polluants susceptibles d'être étudiés, la caractérisation des limites d'Atterberg est une étape préliminaire indispensable à la réalisation d'essais mécaniques de laboratoire visant à étudier l'écrouissage de l'argile polluée.

Enfin, il est notoire que le comportement élastoplastique des argiles présente un lien avec les propriétés physiques [2], [3] and [7] telles que les limites d'Atterberg. Ces limites sont des constantes physiques conventionnelles qui marquent les seuils entre :

- le passage d'un sol de l'état liquide à l'état plastique ( $\underline{W}_{\underline{L}}$ limite de liquidité);

- le passage d'un sol de l'état plastique à l'état solide ( $\underline{W}_{\underline{P}}$ limite de plasticité).

La plupart des travaux de la littérature utilisent la méthode de Casagrande comme une des méthodes standard pour évaluer les propriétés physiques des argiles que sont les limites de consistance. Il est usuellement accepté que les incertitudes sur ces grandeurs atteignent $10 \%$.

Dans ce contexte, où des évolutions significatives des propriétés physiques devraient être observées, une méthodologie de détermination de $\underline{W}_{\underline{L}}$ et $\underline{W}_{\mathrm{P}}$ a été développée. Basé, comme la méthode de Casagrande, sur la mesure de la quantité d'eau libre présente dans un matériau, ce procédé s'appuie sur le phénomène de désaturation du matériau par ventilation dynamique.

Ce procédé présente les avantages suivants :

(i) détermination de $\underline{W}_{\underline{L}}$ et $\underline{W}_{\mathrm{P}}$ en un seul essai ;

(ii) pas d'action humaine directe sur l'échantillon (aucune contrainte mécanique) lors de la transition de phase liquide-solide du matériau.

Il constitue également une solution intéressante pour la détermination des $\underline{W}_{\mathrm{P}}$ des argiles polluées, rendue impossible par la méthode de Casagrande, du fait, lors de la consolidation, de l'écrouissage du matériau.

Ce travail présente les matériaux utilisés : des argiles courantes, la méthode de dessiccation appliquée pour obtenir le passage de la boue à l'état solide, puis la méthodologie de détermination des limites d'Atterberg à partir des grandeurs macroscopiques mesurées.

\section{Matériaux et méthodes}

\subsection{Matériaux}

Les matériaux utilisés pour réaliser cette étude sont des matériaux argileux naturels, à l'état de poudre : 
(i) un kaolin du gisement Quessoy (Côtes-d'Armor), contenant 83 à $89 \%$ de kaolinite, 5 à $7 \%$ de quartz et 7 à $9 \%$ de feldspath et mica ;

(ii) une bentonite sodique du Wyoming appelée MX-80, contenant $88 \%$ de montmorillonite, $4 \%$ de quartz, $2 \%$ de cristobalite, $2 \%$ d'albite et $1 \%$ de calcite ;

(iii) un matériau argileux nommé $\mathrm{Fo}-\mathrm{Ca}$, issu du Bassin parisien et d'âge Sparnacien, constitué de $86 \%$ de minéraux argileux interstratifiés ( $80 \%$ de smectite, $20 \%$ de kaolinite), 6 $\%$ de quartz, $6 \%$ de gœthite, $1,4 \%$ de calcite et $0,4 \%$ de gypse [4].

Les teneurs en eau initiales des boues, $\underline{W}_{\text {i }}$, définies comme le rapport entre la masse d'eau et la masse sèche du matériau mesurée après un passage à l'étuve à $105^{\circ} \mathrm{C}$ pendant $24 \mathrm{~h}$, varient de 74 à $636 \%$ (Tableau 1). Des boues homogènes sont préparées par malaxage mécanique de la poudre d'argile avec de l'eau déminéralisée, de sorte que la teneur en eau initiale soit égale à environ 1,5 fois la limite de liquidité, déterminée par la méthode de Casagrande [2]. Les valeurs de ces constantes sont reportées dans le Tableau 2.

\begin{tabular}{|l|l|l|l|}
\hline & Teneur en eau naturelle $\boldsymbol{W} \mathbf{( \% )}$ & Limite de liquidité $\underline{\underline{W}}_{\underline{\mathbf{L}} \mathbf{~ ( \% )}}$ & Limite de plasticité $\underline{\underline{\underline{\mathbf{P}}}} \mathbf{( \% )}$ \\
\hline Kaolin & 1 & $51 \pm 5$ & $38 \pm 5$ \\
\hline MX-80 & 5,8 & $435 \pm 10$ & $160 \pm 10$ \\
\hline Fo-Ca & 12,5 & $120 \pm 5$ & $51 \pm 5$ \\
\hline
\end{tabular}

Tableau 1. : Propriétés géotechniques des trois matériaux argileux étudiés (limites d'Atterberg déterminées par la technique de Casagrande)

Geotechnical properties of the three studied clay materials (determination of Atterberg limits by Casagrande's method)

\begin{tabular}{|c|c|c|c|c|c|}
\hline & $\begin{array}{l}\text { Teneur en eau de la } \\
\text { boue initiale } \underline{W}_{\underline{i}}(\%)\end{array}$ & $\begin{array}{lr}\text { Limite } & \text { de } \\
\text { liquidité } & \underline{W}_{\mathrm{L}} \\
\text { (\%) } & \end{array}$ & $\begin{array}{lr}\text { Limite } & \text { de } \\
\text { plasticité } & \underline{W_{P}} \\
\text { (\%) } & \end{array}$ & $\begin{array}{l}\text { Teneur en eau de } \\
\text { fissuration } \underline{W}_{f}(\%)\end{array}$ & $\begin{array}{l}\text { Écart } \\
\text { relatif en } \\
\%\end{array}$ \\
\hline Kaolin & 74 & 46 & 42 & 46 & 9,8 \\
\hline $\begin{array}{l}\text { MX- } \\
80\end{array}$ & 636 & 390 & 148 & - & 10,3 \\
\hline $\mathrm{Fo}-\mathrm{Ca}$ & 186 & 118 & 47 & 80 & 1,6 \\
\hline
\end{tabular}

Tableau 2. : Limites d'Atterberg obtenues à partir des essais de dessiccation, teneur en eau à

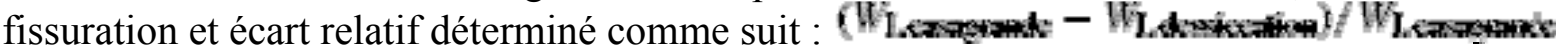

Atterberg limits as identified from desiccation, water content, and relative deviation tests, as follows:

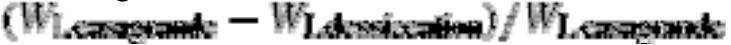




\subsection{Méthode de dessiccation}

La méthode de dessiccation dynamique développée dans cette étude pour déterminer les teneurs en eau, caractéristiques des changements d'état d'échantillons de boues argileuses, est basée sur le principe de transfert de masse en phase vapeur à humidité relative ambiante. La succion est appliquée sur la surface de la face supérieure du matériau contenu dans une cellule verticale, comme le montre la Fig. 1. La cellule, d'une contenance maximale de $250 \mathrm{~g}$ de boue, est constituée d'un cylindre en matériau transparent gradué pour une lecture directe du tassement. Ce cylindre emmanché dans un support est équipé :

(i) à la base, d'une plaque circulaire poreuse en bronze fritté permettant le transfert d'eau en phase vapeur,

(ii) d'un couvercle empêchant une dépression dans la cellule, occasionnée par le départ d'eau au cours de l'essai.

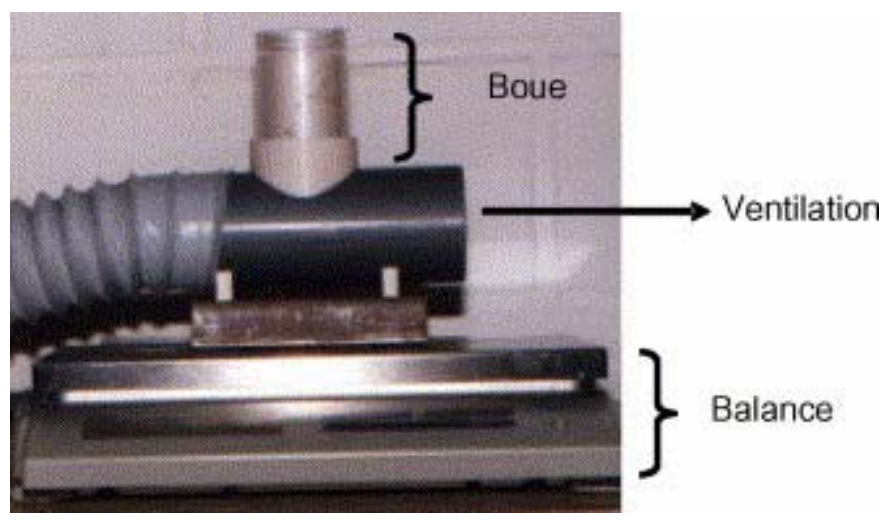

Fig. 1. Appareillage de dessiccation d'une boue.

Equipment used for the experiments of mud dessiccation.

La base de la cellule est raccordée à une veine de dessiccation de $10 \mathrm{~cm}$ de diamètre, dans laquelle circule de l'air à une vitesse de $10 \mathrm{~m} \mathrm{~s}^{-1}$, générée par un ventilateur positionné à l'extrémité du circuit, engendrant une dépression dans la veine. La cellule est positionnée sur une balance d'une précision de $0,01 \mathrm{~g}$, connectée à un ordinateur permettant de mesurer la masse en continu de l'échantillon et d'en déduire une teneur en eau globale instantanée de celui-ci. Le principe des mesures consiste à identifier des points caractéristiques de teneur en eau globale au cours de la dessiccation et de les relier au changement d'état liquide/solide et, par voie de conséquence, aux limites d'Atterberg. Comme les boues sont dé-saturées à volume libre, une fissuration se produit aussi à différentes échelles selon le type de matériaux. Un des points importants de la méthodologie de mesure consistera à vérifier que les mécanismes de fissuration n'altèrent pas la détermination des constantes $\underline{W}_{\underline{L}}$ et $\underline{W}_{\underline{P}}$ par cette méthode.

Après remplissage de la cellule, les expériences sont menées de la façon suivante :

- mesure de la masse en continu et du retrait par lecture du tassement, dès que l'air circule dans la veine de dessiccation; 
- observation visuelle de l'apparition des fissures macroscopiques sur la surface supérieure et latérale de l'échantillon de boue et suivi de leur mode de propagation (radiale ou verticale, en surface et en profondeur);

- arrêt de l'expérience pour une teneur en eau instantanée très inférieure à la limite de plasticité du matériau;

- observation au microscope électronique à balayage d'échantillons de boue séchée, prélevés à proximité de la surface d'échange.

\subsection{Mise au point de la méthodologie de mesure}

L'évolution de la perte de masse et du tassement vertical, obtenus par dessiccation dynamique est tracée en fonction du temps (ig. 2). En choisissant judicieusement l'échelle des axes des ordonnées, les courbes de tassement et de perte de masse se superposent exactement au cours de la première phase de dessiccation, jusqu'à ce que l'argile atteigne sa limite de liquidité. Dans ce domaine, la cinétique de perte de masse et de tassement est due au départ de l'eau libre de la boue.

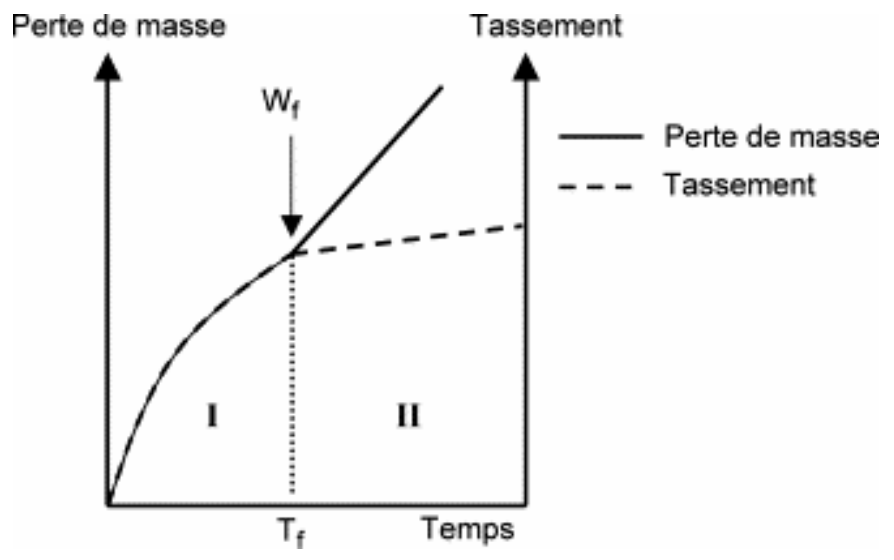

Fig. 2. Courbes types de perte de masse et de tassement vertical en fonction du temps pour une boue. I : boue liquide ; II : boue fissurée.

Mass loss and vertical displacement curves of a mud as a function of time.

Durant la deuxième phase de la dessiccation, le tassement s'effectue toujours plus lentement que la perte de masse d'eau (Fig. 2). Ce brusque changement de comportement s'accompagne, selon les matériaux, de l'apparition d'une fissuration macroscopique. La création de nouvelles surfaces d'échange entraîne alors une accélération du processus de perte de masse par comparaison à celle du tassement. Le point de divergence de ces deux courbes correspond à une teneur en eau notée $\underline{W}_{\mathrm{F}}$.

La teneur en eau instantanée est ensuite calculée pour tous les points, à partir des valeurs de masse mesurées et de la teneur en eau initiale de la boue, puis tracée en fonction du temps (Fig. 3). Pour chaque essai, des tangentes aux courbes teneur en eau-temps sont tracées ; leurs intersections correspondent à des valeurs de teneur en eau particulières, respectivement attribuées aux limites d'Atterberg, $\underline{W}_{\underline{L}}$ et $\underline{W}_{\mathrm{P}}$. 


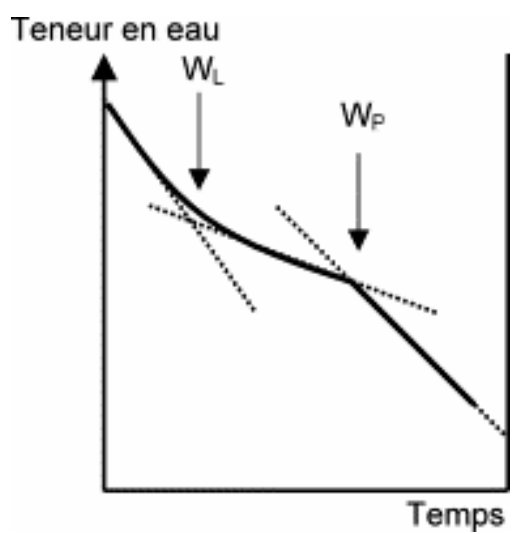

Fig. 3. Evolution type de la teneur en eau instantanée en fonction du temps pour une boue.

Evolution of water content of a mud as a function of time.

\section{Résultats et discussion}

Les résultats obtenus sur les trois matériaux argileux (kaolin, Fo-Ca et Mx-80) sont reportés sur la Fig. 4. Les courbes présentent toutes des discontinuités, attribuées, d'une part, aux limites d'Atterberg $\underline{W}_{\underline{\mathrm{L}}}$ et $\underline{W}_{\mathrm{P}}$ et, d'autre part, à l'apparition de fissures $\underline{W}_{\mathrm{f}}$. Les valeurs de $\underline{W}_{\underline{\mathrm{L}}}$, $\underline{W}_{\mathrm{P}}$ et $\underline{W}_{\mathrm{f}}$ ainsi déterminées sont reportées dans le Tableau 2. 


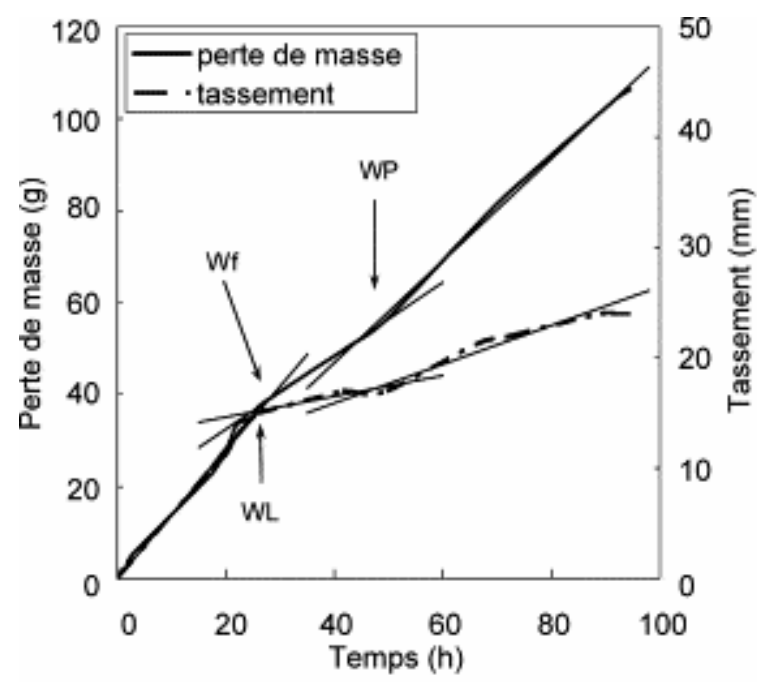

(a)

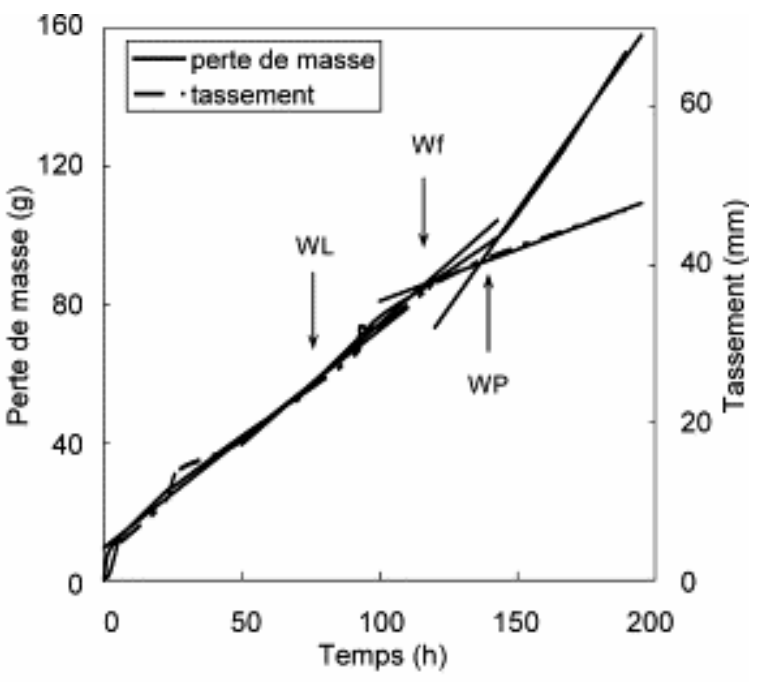

(b)

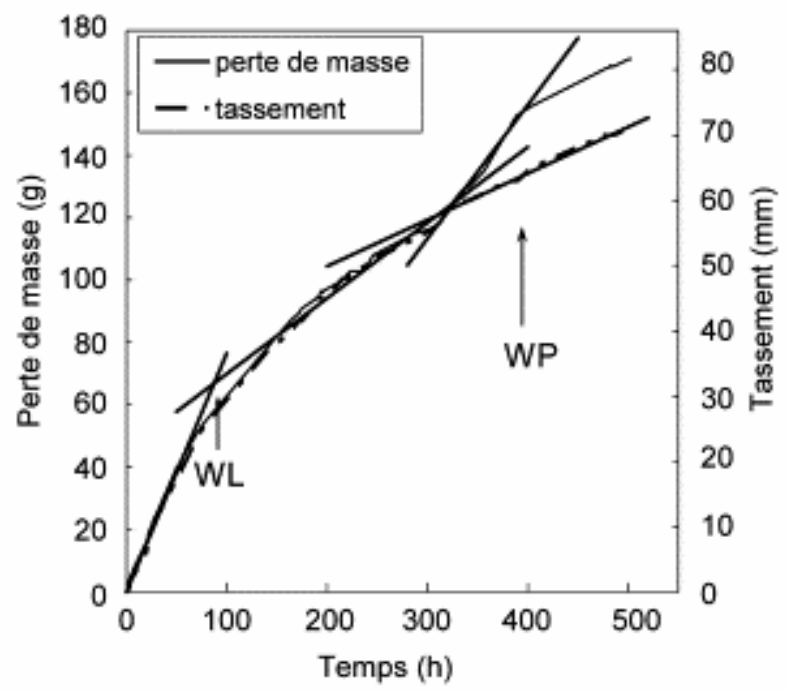

(c)

Fig. 4. Courbes de perte de masse et de tassement vertical en fonction du temps pour une boue de : (a) kaolin, (b) Fo-Ca, (c) MX-80.

Mass loss and vertical displacement curves as a function of time for (a) kaolin mud, (b) FoCa mud, (c) M-80 mud.

L'apparition d'une fissure macroscopique est à noter pour le kaolin et la Fo-Ca. Pour le kaolin, elle apparaît pour une teneur en eau égale à la limite de liquidité, tandis que pour la Fo-Ca, celle-ci est obtenue pour une teneur en eau comprise entre $\underline{W}_{\mathrm{L}}$ et $\underline{W}_{\mathrm{P}}$. Bien que les courbes de perte de masse et de tassement obtenues pour la boue de Mx- 80 présentent une divergence comme dans les cas précédents (Fig. 4c), aucune fissuration n'est décelée à l'échelle macroscopique. L'observation au microscope électronique à balayage en fin d'essais a permis de mettre en évidence un phénomène de fissuration, mais à plus petite échelle (Fig. 5). 


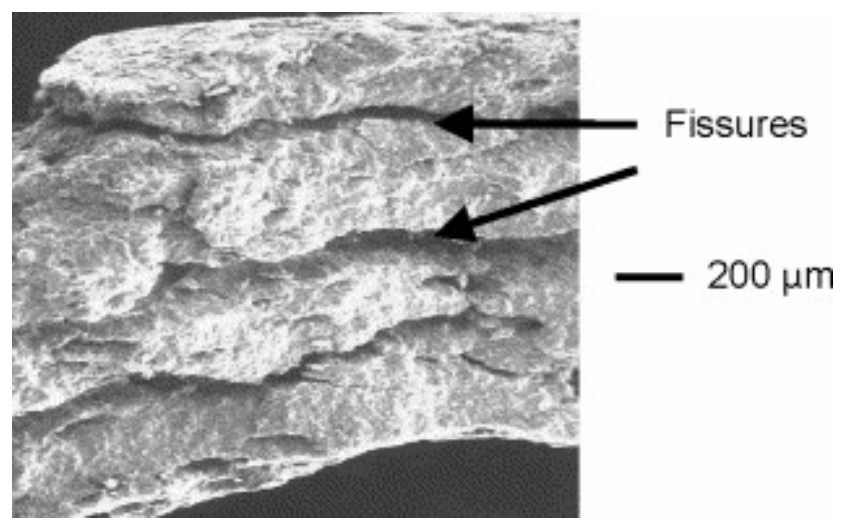

Fig. 5. Cliché de microscopie électronique à balayage obtenu sur l'échantillon de boue de MX-80 après dessiccation.

Scanning electron micrograph of MX-80 mud after desiccation.

Les limites d'Atterberg d'argiles de référence gonflante ou non gonflantes sont aujourd'hui clairement caractérisées par les méthodes classiques, mais les mesures obtenues pour évaluer les propriétés physiques des mélanges d'argiles ou d'argiles polluées posent des problèmes de mise en œuvre, ayant conduit au développement d'une nouvelle méthodologie. Sa mise en œuvre en laboratoire est simple, indépendante du savoir-faire de l'expérimentateur, basée sur des mesures automatisables. Les valeurs de $\underline{W}_{\mathrm{L}}$ et $\underline{W}_{\mathrm{P}}$ (Tableau 2$)$ sont comparées à celles obtenues par la méthode de Casagrande (Tableau 1) : elles sous-estiment ces dernières de 2 à $10 \%$. Une approche similaire a été proposée par Grabowska-Olszewska [3] pour des mélanges d'argiles gonflantes et non gonflantes, comparant des mesures de limite d'Atterberg avec la méthode de Casagrande et celle du cône. Les écarts relatifs entre ces méthodes sont estimés à entre 8 et $15 \%$. D'autre part, différents essais sur le kaolin ont montré que la mesure de $\underline{W}_{\underline{L}}$ et $\underline{W}_{\mathrm{P}}$ était indépendante des fluctuations d'humidité relative de l'air ambiant du laboratoire.

\section{Conclusion}

Ce travail a porté sur la mise au point d'une nouvelle méthodologie de détermination des limites d'Atterberg de boues argileuses par dessiccation dynamique.

Dans tous les cas étudiés, le phénomène de fissuration se produit, mais à des échelles différentes suivant le type de matériau et ne perturbe pas la détermination de $\underline{W}_{\underline{L}}$ et $\underline{W}_{\underline{P}}$. Les mesures effectuées présentent un écart de $10 \%$ au maximum par rapport à celles obtenues par la méthode de Casagrande, permettant d'envisager des développements intéressants de cette méthodologie pour des matériaux pour lesquels cette méthode n'est pas applicable.

\section{Acknowledgments}

Les auteurs remercient Patrick Lecomte, technicien à l'université d'Orléans, pour son assistance technique.

\section{References}


[1] E.E. Alonso, A. Gens and A. Josa, A constitutive model for partially saturated soils, Géotechnique 40 (1990) (3), pp. 405-430.

[2] A. Casagrande, Research on the Atterberg limits of soils, Public roads 13 (1932), pp. 121136.

[3] B. Grabowska-Olszewska, Modelling physical properties of mixture of clays: example of a two-component mixture of kaolinite and montmorillonite, Appl. Clay Sci. 22 (2003), pp. 251-259.

[4] A. Jullien, C. Proust, L. Le Forestier and P. Baillif, Hydro-chemo-mechanical coupling effects on permeability and swelling behaviour of a Ca smectite soaked by $\mathrm{Cu}$ solutions, Appl. Clay Sci. 21 (2002), pp. 143-153.

[5] C. Pothier, Études des propriétés de confinement de matériaux argileux pour barrières ouvragées de stockages de déchets, thèse université d'Orléans, 2001.

[6] J.-C. Robinet, M. Pakzad, A. Jullien and F. Plas, A general modelling of expansive and non expansive clays, Int. J. Numer. Methods Geomech. 23 (1999), pp. 1319-1335.

[7] H.B. Seed, R.J. Woodward Jr. and R. Lundgren, Clay mineralogical aspects of the Atterberg limits, J. Soil Mech. Foundations Division, Proc. Am. Soc. Civil Eng. (1964), pp. $107-131$. 